\title{
PRODUÇ̃̃O E OFERTA DE MUDAS DE ESPÉCIES ARBÓREAS DE USO MÚLTIPLO, DESTINADAS A UNIDADES DE PRODUÇÃO FAMILIAR
}

\author{
Lamartine Soares Bezerra de Oliveira ${ }^{1}$ \\ Leonaldo Alves de Andrade ${ }^{2}$ \\ Ramon Costa Feitosa ${ }^{3}$ \\ Gerlândio Suassuna Gonçalves ${ }^{4}$
}

\section{RESUMO}

O presente trabalho foi desenvolvido no Laboratório de Ecologia Vegetal localizado no Viveiro Florestal do Centro de Ciências Agrárias - UFPB, em Areia - PB, no período de janeiro de 2006 a novembro de 2007. Teve-se como objetivos capacitar agricultores em viveirismo, bem como produzir e distribuir mudas florestais (espécies de uso múltiplo), para cinco comunidades dos municípios de Areia e Alagoa Grande. Com este trabalho foram capacitadas 272 pessoas, em viveirismo, com ênfase em geração de trabalho, renda e inclusão social. A produção e a distribuição de mudas além de envolver diretamente as cinco comunidades, beneficiou também, outras comunidades de diferentes municípios paraibanos, bem como escolas e outras organizações sociais, que tomaram conhecimento das ações e buscaram parcerias. Cerca de 140 mil mudas de 70 espécies florestais de uso múltiplo foram produzidas e distribuídas. Esta iniciativa mostrou que o viveirismo constitui uma atividade viável para a região, sendo capaz de contribuir para a melhoria do meio ambiente, além de gerar trabalho e renda.

Palavras-chave: Viveirismo. Biodiversidade. Agricultura familiar. Inclusão social.

\footnotetext{
${ }^{1}$ Mestrando em Ciências Florestais - Universidade Federal Rural de Pernambuco, soareslt@hotmail.com;

${ }^{2}$ Professor Associado do Laboratório de Ecologia Vegetal - Centro de Ciências Agrárias da Universidade Federal da Paraíba, landrade@cca.ufpb.br;

${ }^{3}$ Graduando em Agronomia - Centro de Ciências Agrárias da Universidade Federal da Paraíba, ramon.costa@hotmail.com;

${ }^{4}$ Mestrando em Agronomia - Centro de Ciências Agrárias da Universidade Federal da Paraíba, gsuassunag@hotmail.com.
} 


\title{
PRODUCTION AND DISTRIBUTION OF SEEDLINGS OF ARBOREAL SPECIES OF USE MULTIPLE FOR A FAMILY PRODUCTION UNITS
}

\begin{abstract}
This study was carried out at the Laboratory of Plant Ecology located in the forestry nursery in the Centro de Ciências Agrárias - UFPB, in Areia City, Paraíba State, from January 2006 to November 2007. It was aimed to improve the abilities of the farmers to practice arboreal nursery, as well as produce and distribute seedlings (multipurpose species) to five communities in the municipalities of Areia and Alagoa Grande. In this work were trained 272 people in the nursery activities, with emphasis on generation of employment, income and social inclusion. The production and distribution of seedlings involved five communities of Areia and Alagoa Grande City, but got benefits to other communities in several municipalities in the regional context, including schools and others social organizations, which are aware of the actions and sought partnerships. Around 140 thousand seedlings of 70 arboreal species of use multiple were produced and distributed. This work showed that forestry nursery is a potential activity for the farmers in this region, being able to contribute to the improvement of the environment, as well to generate employment, income and other social benefits.
\end{abstract}

Keywords: Forestry nursery. Biodiversity. Family agriculture. Social inclusion.

\section{INTRODUÇÃO}

Historicamente tem-se assistido, em nosso país, à exploração dos recursos naturais de forma perdulária, especialmente por empresas que avançam com os monocultivos, como a pecuária, ou mesmo com a urbanização.

A abertura de estradas e a execução de grandes projetos agrominerais e hidroelétricos, além da comercialização em larga escala de diversas espécies de interesse econômico, são atividades que igualmente respondem pela devastação florestal (ANDRADE et al., 2007).

A exploração e uso dos recursos florestais afetam perigosamente a biodiversidade (LUGO, 1995), a qualidade do ar (MAZZONI VIVEIROS, 2000), da água (EYSINK \& MORAES, 1998; BERTOLETTI, 2001), dos solos (DIAS \& GRIFFITH, 1998) e, consequentemente, a sobrevivência futura de todas as espécies. 
Diante desta realidade, Galvão (2002) relata que o Brasil se apresenta como um dos países com maior índice de desmatamento. Embora, a mídia enfoque, especialmente, as questões amazônicas, o problema é comum a todas as regiões brasileiras. Os Estados da região Nordeste, já perderam parte expressiva de sua cobertura florestal nativa, e estima-se que mais de $70 \%$ dos remanescentes se encontram com algum grau de antropização (SNE, 2002; MMA, 2002). Dentre os inúmeros problemas causados pela devastação florestal ressalta-se a extinção de espécies, a perda de solos, a escassez dos recursos hídricos e, consequentemente, o empobrecimento das populações atingidas.

No Brasil, a pobreza rural concentra-se principalmente na região Nordeste. As unidades de produção familiar aí inseridas apresentam um conjunto de vulnerabilidades que fragilizam os sistemas produtivos. Neste contexto os recursos florestais ainda existentes vêm sendo explorados de forma inadequada.

A solução para este problema exige mudanças coletivas de posturas, hábitos e estratégias de exploração e gestão dos recursos naturais. A silvicultura conservacionista representa parte da solução para esta situação, seja pela lista de produtos que oferece, seja pela função ambiental que a vegetação desempenha.

O presente trabalho objetivou difundir o viveirismo e a silvicultura conservacionista a partir da capacitação técnica respaldada numa vivência prática, que envolveu o público beneficiário, resultando na oferta de cursos, com ênfase na coleta de sementes, na produção de mudas, na distribuição e no plantio de espécies florestais de uso múltiplo, em comunidades rurais do brejo paraibano.

\section{MATERIAL E MÉTODOS}

O trabalho foi desenvolvido entre janeiro 2006 e novembro de 2007 pela equipe do Laboratório de Ecologia Vegetal, do Centro de Ciências Agrária (CCA), da Universidade Federal da Paraíba (UFPB). O público alvo eram pessoas pertencentes a cinco comunidades rurais localizadas nos municípios paraibanos de Areia e Alagoa Grande, para as quais o trabalho foi direcionado, visando à melhoria do meio ambiente e da qualidade de vida dos beneficiários.

Inicialmente foram realizadas reuniões nas cinco comunidades alvos: Projeto de Assentamento (PA) Socorro, PA Margarida Maria Alves II, PA Professor José Horácio, PA 
Maria da Penha II e PA União, a fim de divulgar as ações do projeto, levantar a lista das espécies nativas e de uso múltiplo de interesse dos agricultores e selecionar grupos de agricultores e jovens para serem capacitados em viveirismo.

O projeto teve duas metas, a primeira meta: oferta de cursos para capacitação de 100 agricultores em atividades de viveirismo. Esta etapa, a fim de contribuir com a difusão do viveirismo, como uma atividade capaz de melhorar o meio ambiente, gerando trabalho, renda e a inclusão social, foi dividida nos dois seguintes módulos: Módulo I - Coleta, beneficiamento e armazenamento de sementes de espécies florestais e; Módulo II - Formação de viveiros e produção de mudas florestais, Ambos os módulos foram ministrados nos finais de semana, visando facilitar a participação dos agricultores.

Após a inscrição dos participantes e a entrega do material didático, iniciavam-se as atividades com uma dinâmica de apresentação e integração da equipe com os participantes. Pela manhã, iniciava-se a exposição teórica do conteúdo técnico, de forma ilustrada e participativa, com quatro horas aulas intercaladas, com intervalos para lanche e troca de experiências. À tarde, realizavam-se as práticas de viveirismo no viveiro florestal do CCA/UFPB, com visitas aos Laboratórios de Ecologia Vegetal e de Análise de Sementes.

A segunda meta, a produção e distribuição de 130 mil mudas florestais e de uso múltiplo, a qual iniciou com expedições de coleta de sementes nos remanescente florestais, ainda, existentes nas comunidades beneficiadas e nas adjacências do CCA/UFPB. Algumas sementes das espécies solicitadas pelos agricultores foram adquiridas através de compra devido à sua inexistência ou insuficiência na região. Alguns exemplos dessas espécies são sementes de sabiá, cedro, jatobá e algumas frutíferas como tamarindeiro e cajueiro precoce.

Todos os frutos e sementes coletados foram beneficiados nas próprias dependências do Laboratório de Ecologia Vegetal. As sementes foram separadas dos frutos através de técnicas de lavagens em água corrente, uso de despolpadeira, de tamises e, para algumas espécies, através do beneficiamento manual. A separação de sementes e de impurezas se fez com o uso de peneiras de diferentes malhas e a secagem, realizada a pleno sol ou à sombra. Sementes de algumas espécies foram armazenadas em câmara fria logo após o beneficiamento, acondicionadas em sacolas, garrafas plásticas e em recipientes impermeáveis, garantindo com isto a viabilidade das mesmas em todas as etapas do projeto.

A produção das mudas foi realizada por meio do semeio direto nos recipientes (de polietileno preto), uso de sementeiras com posterior transplante das mudas para os recipientes e propagação clonal ou vegetativa, empregando-se estacas lenhosas e semilenhosas. 


\section{RESULTADOS E ANÁLISE}

\subsection{CURSOS DE CAPACITAÇÃO EM VIVEIRISMO}

Os cursos de capacitação em viveirismo que envolveram a coleta, o beneficiamento e o armazenamento de sementes florestais, bem como todas as etapas necessárias para produção e condução de mudas florestais em viveiro contribuíram para a difusão do viveirismo na região, uma vez que geraram trabalho e renda para a população, assim como promoção de melhoria da qualidade ambiental. Os cursos superaram todas as expectativas, sendo capacitadas ao final do projeto 272 pessoas, ultrapassando o previsto, inicialmente, no Projeto (Tabela 1).

Tabela 1. Relação dos cursos de capacitação em viveirismo promovidos no Centro de Ciências Agrárias da UFPB, no período de agosto a novembro de 2006.

\begin{tabular}{lcccc}
\hline \multicolumn{1}{c}{ Cursos de Capacitação em Viveirismo } & Módulo & $\begin{array}{c}\text { Número de } \\
\text { Participantes }\end{array}$ & Datas \\
\hline $\begin{array}{l}\text { Coleta, Beneficiamento e Armazenamento de } \\
\text { Sementes Florestais. }\end{array}$ & I & 21 & $05 / 08 / 2006$ \\
$\begin{array}{l}\text { Coleta, Beneficiamento e Armazenamento de } \\
\text { Sementes Florestais. }\end{array}$ & I & 50 & $19 / 08 / 2006$ \\
$\begin{array}{l}\text { Coleta, Beneficiamento e Armazenamento de } \\
\text { Sementes Florestais. }\end{array}$ & I & 54 & $26 / 08 / 2006$ \\
$\begin{array}{l}\text { Coleta, Beneficiamento e Armazenamento de } \\
\text { Sementes Florestais. }\end{array}$ & I & 26 & $02 / 09 / 2006$ \\
$\begin{array}{l}\text { Formação de Viveiros e Produção de Mudas } \\
\text { Florestais. }\end{array}$ & II & 32 & $07 / 10 / 2006$ \\
$\begin{array}{l}\text { Formação de Viveiros e Produção de Mudas } \\
\text { Florestais. }\end{array}$ & II & 24 & $14 / 10 / 2006$ \\
$\begin{array}{l}\text { Formação de Viveiros e Produção de Mudas } \\
\text { Florestais. }\end{array}$ & II & 44 & $21 / 10 / 2006$ \\
$\begin{array}{l}\text { Formação de Viveiros e Produção de Mudas } \\
\text { Florestais. }\end{array}$ & II & 21 & $04 / 11 / 2006$ \\
\hline Total & - & 272 & \\
\hline
\end{tabular}

Este trabalho promoveu, também, uma aproximação do público alvo com a Universidade, representando uma realidade antes não vista, embora estas comunidades se localizem na mesma região do Campus universitário.

O conteúdo abordado no primeiro módulo teve como principal enfoque a recuperação e a conservação dos remanescentes florestais da região, como meio de melhorar a qualidade PRODUÇÃO E OFERTA DE MUDAS DE ESPÉCIES ARBÓREAS DE USO MÚLTIPLO, DESTINADAS A UNIDADES DE PRODUÇÃO.

Oliveira, L.S.B.; Andrande, L.A; Feitosa, R.C;Gonçalves, G.S. 
de vida dos beneficiários e como fontes de obtenção de sementes não somente para produção de mudas, mas também para a obtenção de sementes destinadas a venda e ao artesanato.

Ainda no primeiro módulo foram realizadas demonstrações práticas de como selecionar árvores matrizes para a obtenção de sementes; os métodos e as técnicas de se coletar sementes florestais; como classificar as sementes; como beneficiar e secar as sementes florestais e, por fim, as condições de armazenamento, os tipos de embalagens e os tratamentos convencionais e alternativos para conservação de sementes florestais (Figura 1).
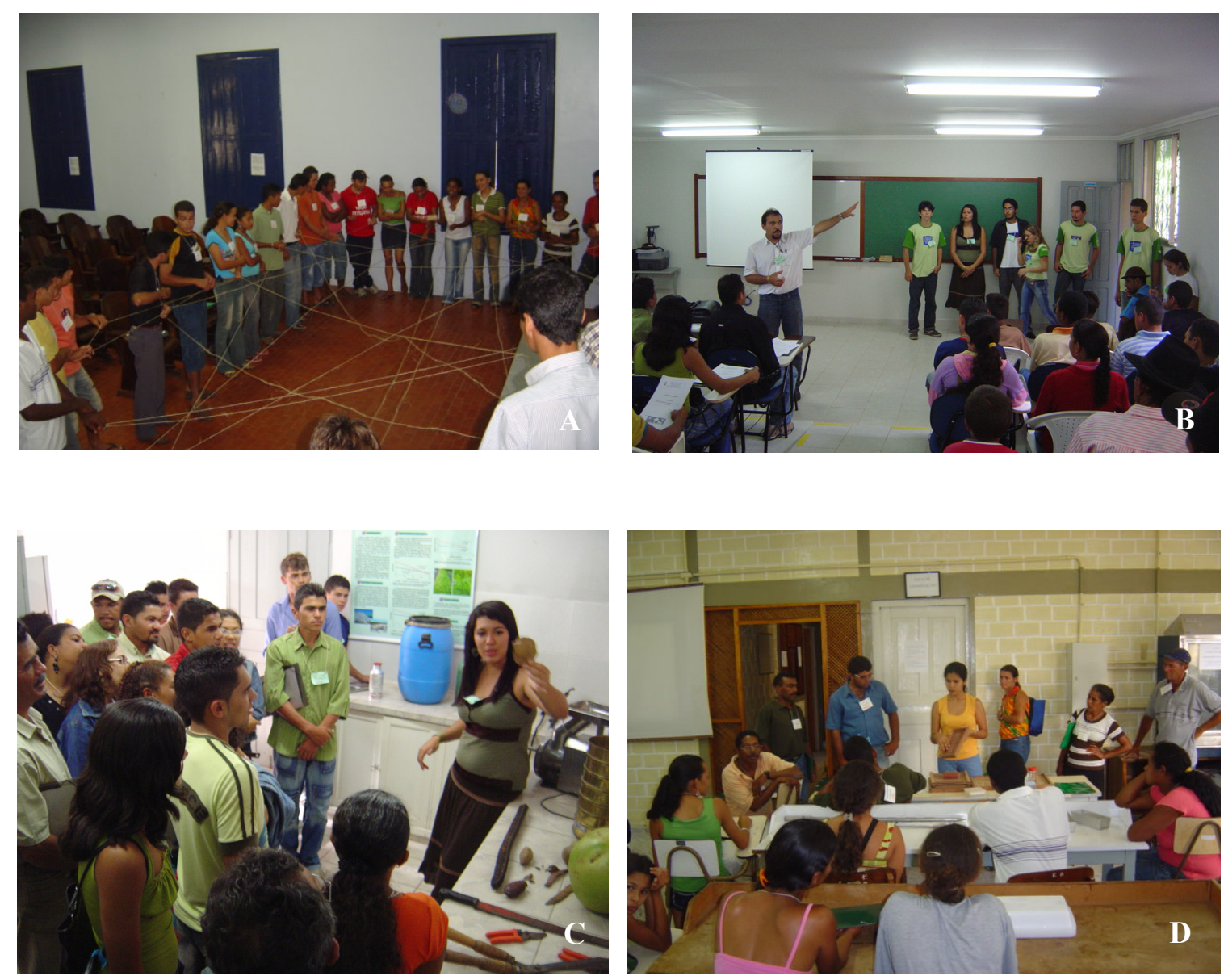

Figura 1. Participantes do Módulo I dos cursos de capacitação em viveirismo: dinâmica de integração (A); aula teórica (B); aula prática no Laboratório de Ecologia Vegetal (C) e no Laboratório de Análise de Sementes (D), no CCA/UFPB.

Nos conteúdos teóricos e na abordagem prática do segundo módulo (Figura 2) foram direcionados os conhecimentos necessários para formação de viveiros e a produção de mudas florestais. Foram apresentadas as estruturas comumente encontradas em viveiros convencionais, alternativos, temporários ou permanentes. Também foram trabalhados aspectos ligados à produção, a distribuição e a comercialização de mudas, bem como a 
localização; a infraestrutura mínima necessária de um viveiro; o dimensionamento de um viveiro; a construção de canteiros; o uso e a confecção de sementeiras; os tipos de substratos (convencionais e alternativos); os recipientes utilizados na produção de mudas; os métodos de produção de mudas; a avaliação da qualidade das mudas; a comercialização e o plantio de mudas florestais e de uso múltiplo.
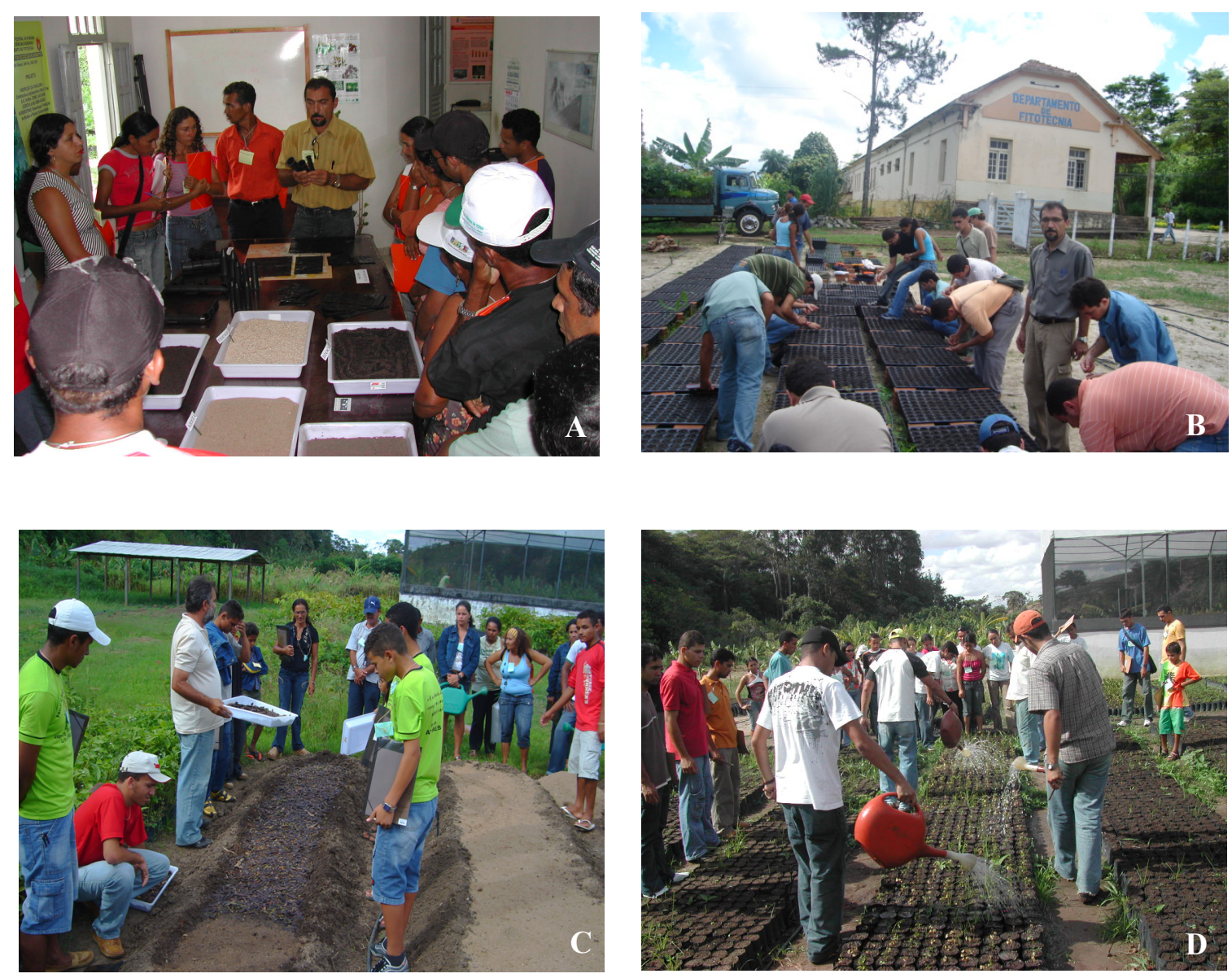

Figura 2. Participantes do Módulo II dos cursos de capacitação em viveirismo nas aulas práticas realizadas no viveiro florestal do CCA/UFPB: apresentação dos tipos de substratos e recipientes utilizados na produção de mudas (A); semeadura direta em recipientes (B); construção de sementeira $(\mathrm{C})$; rega de mudas recém transplantadas $(\mathrm{D})$.

\subsection{PRODUÇÃO E DISTRIBUIÇÃO DAS MUDAS}

Durante todas as etapas do processo de produção de mudas, os beneficiários visitaram o viveiro com o objetivo de conhecer e acompanhar o trabalho. Inicialmente, estas visitas foram programadas apenas para as comunidades beneficiadas, mas diante da divulgação do 
Projeto, várias escolas municipais, estaduais e particulares da região, bem como a Universidade Estadual Paraíba e a Federal de Campina Grande organizaram grupos de estudantes para conhecer o trabalho (Figura 3).
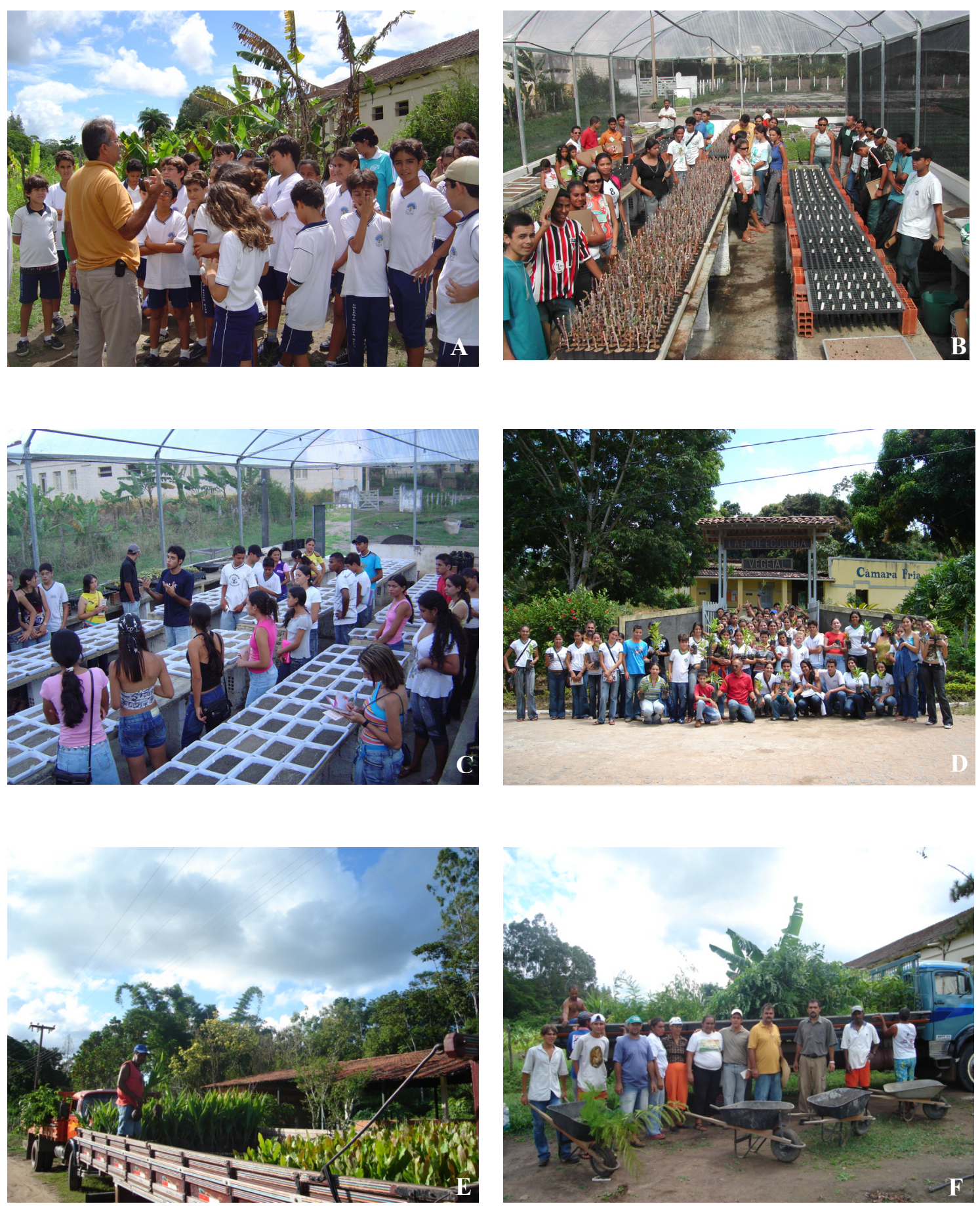

Figura 3. Visitas realizadas por agricultores e estudantes para conhecer as atividades de produção de mudas realizadas pelo Laboratório de Ecologia Vegetal no viveiro florestal do CCA/UFPB (A, B, C e D); e distribuição das mudas para as comunidades rurais $(\mathrm{E}$ e $\mathrm{F})$.

PRODUÇÃO E OFERTA DE MUDAS DE ESPÉCIES ARBÓREAS DE USO MÚLTIPLO, DESTINADAS A UNIDADES DE PRODUÇÃO.

Oliveira, L.S.B.; Andrande, L.A; Feitosa, R.C; Gonçalves, G.S. 
No total foram produzidas cerca de 140 mil mudas, ou seja, 10 mil mudas a mais que o previsto, inicialmente. As mudas produzidas contemplaram 70 espécies florestais de uso múltiplo, muitas das quais são de grande importância e já não existiam mais nas comunidades. Como exemplo destas espécies, temos baraúna, cedro, jacarandá, maçaranduba, pau-brasil, pau-ferro dentre outras (Tabela 2).

Tabela 2. Espécies florestais e de uso múltiplo e respectivas quantidades de mudas produzidas no âmbito da execução do Projeto, no viveiro florestal do CCA/UFPB.

\begin{tabular}{|c|c|c|}
\hline Espécie & Nome Vulgar & Qtde. \\
\hline Euterpe oleracea Mart. & Açaí & 100 \\
\hline Mimusops elengi L. & Abricó-do-mato & 2.300 \\
\hline Malpighia glabra L. & Acerola & 50 \\
\hline Anadenanthera macrocarpa Benth. & Angico & 2.300 \\
\hline Annona muricata $\mathrm{L}$. & Araticum & 120 \\
\hline Schinus molle L. & Aroeira & 200 \\
\hline Carapa guianensis Aubl. & Andiroba & 20 \\
\hline Bambusa arundinacea (Retz.) Willd. & Bambu-ornamental & 120 \\
\hline Dendrocalamus strictus (Roxb.) Nees & Bambu-gigante & 80 \\
\hline Schinopsis brasiliensis Engl. & Baraúna & 320 \\
\hline Anacardium occidentale L. & Cajueiro & 20.000 \\
\hline Tabebuia caraíba (Mart.) Bur. & Craibeira & 23.000 \\
\hline Thevetia ahouai (L.) A. DC. & Chapéu-de-napoleão & 150 \\
\hline Caesalpinia brasiliensis L. & Cássia-brasil & 1.280 \\
\hline Caesalpinia sp. & Cássia-ferruginea & 700 \\
\hline Cássia grandis L.F. & Canafístula & 390 \\
\hline Pachira aquática Aubol. & Castanha-do-maranhão & 100 \\
\hline Cedrela odorata $\mathrm{L}$. & Cedro & 1.000 \\
\hline Senecio douglasii DC. & Cinerária & 200 \\
\hline Cocos nucifera $\mathrm{L}$. & Coco & 1.700 \\
\hline Eucaliptus sp. & Eucalipto & 800 \\
\hline Cnidosculus phyllacanthus (Mart.) Pax. et K. Hoffm. & Faveleira & 300 \\
\hline Balizia pedicellaris (DC.) Barneby \& J.W. Grimes & Faveira-roxa & 400 \\
\hline Parkia paraensis Ducke & Faveira-branca & 200 \\
\hline Ficus sp. & Fícus & 150 \\
\hline Delonix regia (Bojer ex Hook.) Raf. & Flamboyant & 450 \\
\hline Gliricídia sp. & Gliricidia & 250 \\
\hline Schizolobium parahiba Vell & Guapuruvu & 100 \\
\hline Inga ingoides (Rich.) Willd. & Ingá & 100 \\
\hline Tabebuia uliginosa (Gomes) A. DC. & Ipê-amarelo & 2.000 \\
\hline \multirow[t]{2}{*}{ Tabebuia impetigiosa sp. } & Ipê-roxo & 100 \\
\hline & & Continua \\
\hline
\end{tabular}


Tabela 2. Cont.

\begin{tabular}{|c|c|c|}
\hline Espécie & Nome Vulgar & Qtde. \\
\hline Tabebuia avellanedae Lor.ex.Griseb & Ipê-rosa & 11.000 \\
\hline Tabebuia sp. & Ipê-branco & 1.000 \\
\hline Myrcia cauliflora Berg & Jabuticaba & 1.900 \\
\hline Dalbergia nigra (Vell.) Allemão ex Benth. & Jacarandá & 30 \\
\hline Artocarpus sp. & Jaca & 250 \\
\hline Syzygium malaccense (L.) Merr. \& L.M. Perry. & Jambo & 7.000 \\
\hline Hymenaea courbaril L. & Jatobá & 3.000 \\
\hline Genipa americana $\mathrm{L}$. & Jenipapo & 1.200 \\
\hline Zizyphus joazeiro Mart. & Juá & 300 \\
\hline Aspidosperma sp. & Peroba & 300 \\
\hline Lophantera lactescens sp. & Lanterneiro & 150 \\
\hline Leucaena leucocephala Lam. & Leucena & 450 \\
\hline Manilkara kauki (L.) Dubard & Maçaranduba & 20 \\
\hline Moringa oleifera Lam. & Moringa & 1.100 \\
\hline Erythrina velutina Willd. & Mulungu & 600 \\
\hline Azadirachta indica A. Juss. & Nim & 900 \\
\hline Licania tomentosa Benth. & Oiti & 4.000 \\
\hline Areca catechu L. & Palmeira-areca & 800 \\
\hline Roystonea regia (Kunth) & Palmeira-imperial & 30 \\
\hline Caryota gigas & Palmeira-molambo & 80 \\
\hline Roystonea oleracea sp. & Palmeira-de-vaso & 2.700 \\
\hline Bauhinia aculeata L. & Pata-de-vaca & 400 \\
\hline Caesalpinia echinata Lan & Pau-brasil & 2.200 \\
\hline Gallesia integrifólia (Spreng.) Harms & Pau-d`alho & 30 \\
\hline Chamaecrista nictitans (L.) Moench & Pau-ferro & 20 \\
\hline Aspidosperma spp. & Pereiro & 420 \\
\hline Duranta erecta L. & Pingo-de-ouro & 5.000 \\
\hline Annona squamosa $\mathrm{L}$. & Pinha & 3.000 \\
\hline Eugenia uniflora L. & Pitanga & 800 \\
\hline Pinus sp. & Pinheiro & 20 \\
\hline Mimosa caesalpiniifolia Benth. & Sabiá & 30.000 \\
\hline Clitoria fairchildiana Howard & Sombreiro & 20 \\
\hline Pseudobombax marginatum (A. St. Hil.) A. Robyns & Sumaúma & 70 \\
\hline Bowdichia virgilioides Kunth. & Sucupira & 1.000 \\
\hline Phoenix dactylifera $\mathrm{L}$. & Tâmara & 100 \\
\hline Tamarindus indica L. & Tamarindo & 100 \\
\hline Enterolobium contortisiliquum (Vell.) Morong & Tambor & 400 \\
\hline Spondias tuberosa Arruda Câm. & Umbu & 20 \\
\hline Bixa orelana L. & Urucum & 450 \\
\hline Total & - & 139.850 \\
\hline
\end{tabular}

Todas as mudas produzidas, inicialmente, seriam destinas ao plantio apenas nas cinco comunidades de Areia e Alagoa Grande, porém devido aos resultados iniciais obtidos pelo Projeto, várias comunidades de diferentes municípios paraibanos (Alagoa Nova, Arara, PRODUÇÃO E OFERTA DE MUDAS DE ESPÉCIES ARBÓREAS DE USO MÚLTIPLO, DESTINADAS A UNIDADES DE PRODUÇÃO.

Oliveira, L.S.B.; Andrande, L.A; Feitosa, R.C;Gonçalves, G.S. 
Araruna, Areial, Bananeiras, Belém, Dona Inês, Gurinhém, Jacaraú, Mari, Pilões, Puxinanã, Remígio, Sertãozinho, e Solânea, dentre outros), também foram contemplados com mudas, mediante solicitações.

\section{CONSIDERAÇÕES FINAIS}

- As estratégias metodológicas utilizadas motivaram os participantes, mostrando a necessidade e a pertinência de se plantar e cultivar árvores, seja com fins conservacionistas, seja com fins econômicos;

- A forma como os cursos foram ministrados motivou os agricultores, de forma que a procura pelos cursos superou todas as expectativas inicialmente previstas;

- Ao longo deste trabalho foram produzidas cerca de 140 mil mudas de espécies arbóreas nativas e de uso múltiplo, as quais foram distribuídas e plantadas nas comunidades, trazendo benefícios ambientais e socioeconômicos;

- Observou-se que a demanda por mudas foi maior que a oferta, ratificando a certeza de que o viveirismo pode tornar-se uma alternativa rentável para as comunidades rurais no contexto regional;

- Estão surgindo iniciativas de produção de mudas por parte dos agricultores em viveiros rústicos instalados por eles próprios, visando aplicar os conhecimentos adquiridos e incorporar o viveirismo como mais uma atividade promissora à realidade socioambiental por eles vivenciada, o que mostra a tendência de continuidade das ações;

- Por fim, este trabalho mostrou que o viveirismo constitui uma atividade viável para a região e que tem boa aceitação pelos agricultores. Desta forma, vislumbra-se não só a melhoria das condições ambientais, mas também a geração de trabalho e renda a partir desta vertente, o que, aliás, constituiu um dos objetivos desta proposta.

\section{AGRADECIMENTOS}

Os autores agradecem ao CT-AGRO/CT-HIDRO/MCT/CNPq pelo apoio financeiro concedido ao Projeto. Agradecem igualmente aos agricultores e demais pessoas que participaram deste trabalho, mostrando a pertinência da proposta e contribuindo para a difusão 
da silvicultura conservacionista, capaz de promover benefícios ambientais, sociais e econômicos.

\section{REFERÊNCIAS}

ANDRADE, L. A., OLIVEIRA, L. S. B., VIEIRA, R. M., GONÇALVES, G. S. Viveirismo para agricultores familiares: uma iniciativa capaz de gerar trabalho e renda, além de promover a inclusão social. Extensão cidadã - Revista Eletrônica de extensão da UFPB, Ano I, n. 01, janeiro/junho de 2006.

BERTOLETTI, E. Ecotoxicologia aquática. In: MAIA, N. B. et al. Indicadores ambientais: conceitos e aplicações. São Paulo. Educ. 2001.

DIAS, L. E.; GRIFFITH, J. J. Conceituação e caracterização de áreas degradadas. In: MELLO, J. W. W. (Ed.). Recuperação de áreas degradadas. Viçosa, MG: Universidade Federal de Viçosa, 1998, 17p.

EYSINK ,G. G. J.; MORAES, R. P. Subsídios para manejo e recuperação de ecossistemas aquáticos contaminados por metais pesados. In: MELLO, J. W. W. (Ed.). Recuperação de áreas degradadas. Viçosa, MG: Universidade Federal de Viçosa, 1998, p. 235 - 246.

GALVÃO, A. P. M. (Org.) Reflorestamento de propriedades rurais para fins produtivos e ambientais. EMBRAPA, Brasília, 2000, 351 p.

LUGO, E. A. Managementt of tropical biodiversity. Ecologycal Applicatons, New York, v. 5, n. 4, p. 956 - 959, 1995.

MAZZONIVIVEIROS, S. C. Diversidade do uso da anatomia: poluição atmosférica na Mata Atlântica. In: SAVALCANTE, T. B. et al. (Org.). Tópicos atuais em botânica: palestras convidadas do $51^{\circ}$ Congresso Nacional de Botânica. Brasília: Embrapa, Sociedade Botânica de Brasil, p. 101- 106, 2000.

MMA - Ministério do Meio Ambiente, dos Recursos Hídricos e da Amazônia Legal. AVALIAÇÃO e ações prioritárias para a conservação da biodiversidade da caatinga/ por: Universidade Federal de Pernambuco/Fundação de apoio ao desenvolvimento, Fundação Biosiversitas, EMBRAPA/Semi-Árido, Brasil: MMA/SBF, 2002. 36p.

SOCIEDADE NORDESTINA DE ECOLOGIA. Mapeamento da Mata Atlântica, seus ecossistemas associados: Paraíba e Rio Grande do Norte, Relatório Técnico, 2002. 